\title{
Locality-Aware Network Utilization Balancing in NoCs
}

\author{
ANKIT MORE and BARIS TASKIN, Drexel University
}

Hierarchical and multi-network networks-on-chip (NoCs) have been proposed in the literature to improve the energy- and performance-efficient scalability of the traditional flat-mesh NoC architecture. Theoretically, based on a small-world network-based analysis, traditional hierarchical NoCs are expected to provide good scalability. However, the traditional theoretical analysis (e.g. for small-worldness) does not take into account the congestion phenomenon experienced in such networks. Counterintuitively, as shown in this work, breaking the hierarchy in traditional hierarchical NoCs and utilizing the proposed locality-aware network utilization (NU) balancing technique performs better. This improvement in performance is observed through experimental analysis, which is contrasted with the theoretical analysis that does not account for congestion. In addition to the novelties for hierarchical networks, the application of the proposed locality-aware NU balancing scheme is extended to multi-network NoC topologies (with already separated networks). Results of the analysis show the superiority of applying the locality-aware NU balancing technique for a throughput and energy-efficient scaling of the multi-network NoC architectures, much like those of the hierarchical NoCs. For instance, for a NoC with 1024 nodes, the proposed NU balancing technique provides up to 95\% higher throughput efficiency and consumes up to $29 \%$ less energy per flit compared to the best NoC topology without the NU balancing technique. The analysis also helps to render the choice of a NoC topology for traffic patterns varying in locality and nonlocality on exascale computing CMPs.

Categories and Subject Descriptors: B.4.3 [Input/Output and Data Communications]: Interconnections (subsystems)

General Terms: Design, Algorithms, Performance

Additional Key Words and Phrases: Network-on-chip (NoC), hierarchical networks, network utilization balancing

\section{ACM Reference Format:}

Ankit More and Baris Taskin. 2015. Locality-aware network utilization balancing in NoCs. ACM Trans. Des. Autom. Electron. Syst. 21, 1, Article 6 (November 2015), 26 pages.

DOI: http://dx.doi.org/10.1145/2743012

\section{INTRODUCTION}

The demand for high performance computing has fueled the integration of hundreds of computational and storage cores on to a single chip multiprocessor (CMP) [Borkar 2007]. As the size of the CMP increases, the design of the communication fabric plays a critical role in defining the performance and energy consumption of the overall system [Marculescu et al. 2009]. Furthermore, achieving exascale performance under an affordable power budget requires a tremendous amount of parallelism, thereby placing increasingly stringent energy and performance constraints on the communication fabric [Kogge et al. 2008]. It is shown in Balfour and Dally [2006], that a flat-mesh

This material is based upon work supported by the National Science Foundation under Grants No. EECS1232164, CNS-1004936 and CNS-1305350.

Authors' addresses: Department of Electrical and Computer Engineering, Drexel University, Philadelphia, PA 19104; corresponding author email: ankitmore@gmail.com.

Permission to make digital or hard copies of part or all of this work for personal or classroom use is granted without fee provided that copies are not made or distributed for profit or commercial advantage and that copies show this notice on the first page or initial screen of a display along with the full citation. Copyrights for components of this work owned by others than ACM must be honored. Abstracting with credit is permitted. To copy otherwise, to republish, to post on servers, to redistribute to lists, or to use any component of this work in other works requires prior specific permission and/or a fee. Request permissions from permissions@acm.org. (c) 2015 ACM 1084-4309/2015/11-ART6 $\$ 15.00$

DOI: http://dx.doi.org/10.1145/2743012 


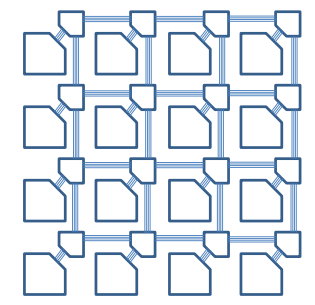

(a) Flat-mesh.

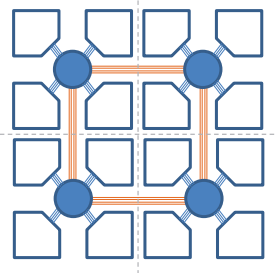

(b) CMesh.

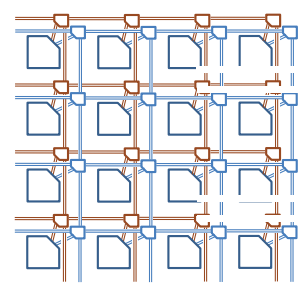

(c) MeshX2.

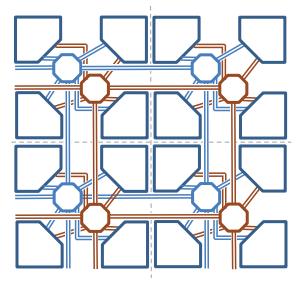

(d) CMeshX2.

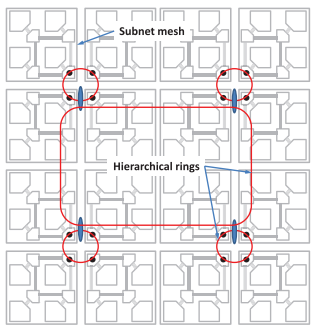

(e) Mesh-rings.



(f) Mesh-mesh.

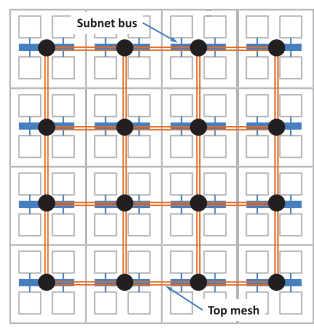

(g) Bus-mesh.

Fig. 1. Single (a, b), multi-network (c, d), and hierarchical (e, f, g) NoCs.

(Figure 1(a)) is not scalable for large CMPs due to its rapidly growing diameter, which results in a higher hop count, thereby increasing the latency and power consumption. To counter the limitations of the flat-mesh, various hierarchical and multi-network network-on-chip (NoC) topologies are proposed in Balfour and Dally [2006], Bourduas and Zilic [2007], and Das et al. [2009], some of which are depicted in Figure 1.

The hierarchical NoCs (depicted in Figures 1(e) through 1(g)) partition a large ubiquitous topology, such as a mesh, into smaller subnets of cores and in-turn connect the subnet of cores at the top level network topology, such as a mesh or a ring topology. On the other hand, multi-network NoCs (depicted in Figures 1(c) and 1(d)) proposed in Balfour and Dally [2006] and Yoon et al. [2010] employ isolated networks serving the same processing elements to address performance scalability through hardware redundancy. The hierarchical structures proposed in Balfour and Dally [2006], Bourduas and Zilic [2007], and Das et al. [2009] are effective in reducing the network diameter (as indicated by the low average internode distance and high clustering coefficient properties discussed in Section 6). However, the cores in a single subnet of these hierarchical structures are only allowed to communicate outside the subnet (inter-subnet traffic) through a single access point. This incumbent hierarchy of subnets operating in isolation, which is referred to as hard-walled core subnets in this article, creates a bottleneck in accessing the top level network due to a greater contention for limited resources (a phenomenon not captured by the small-world-like metrics discussed in Section 6). Therefore, despite enjoying lower latencies, the hierarchical NoCs have a poor throughput, as also discussed in Das et al. [2009]. In this work, these hard-walled core subnets are broken in order to establish the proposed leaky-walled core subnets for superior exascale CMP performance. Furthermore, a locality-aware network utilization balancing technique is proposed to utilize the leaky-walled subnets more efficiently.

Similarly, the multi-network NoCs also have isolation, not on subnets but between the networks, where the CMP performance depends on the efficient utilization of the redundant resources. The proposed locality-aware network utilization balancing scheme also addresses this efficient-scaling challenge of multi-network NoCs. To this end, the first two novelties of this work are in improving the energy- and performance-efficient 


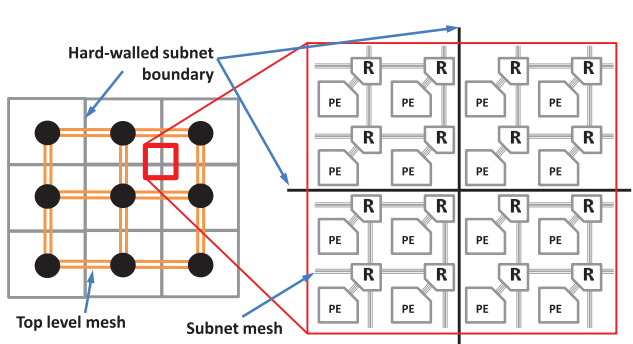

(a) With hard-walled subnets.

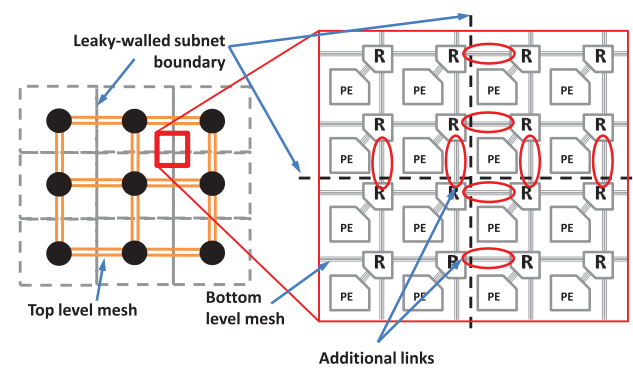

(b) With leaky-walled subnets.

Fig. 2. Breaking the hierarchy in the traditional mesh-mesh NoC topology.

scaling of hierarchical and multi-network NoC topologies by utilizing the existing resources more efficiently, as introduced in Section 1.1. The third novelty of this work is in evaluating the small-world-like properties of such complex networks, as introduced in Section 1.2.

\subsection{Hierarchical and Multi-Network NoC Topologies}

This work proposes a throughput-efficient and energy-efficient design of NoCs for future CMPs by the following.

(I) Breaking the hierarchy in hierarchical NoCs using leaky-walled subnets. The throughput limitation of traditional hierarchical NoCs due to the hard-walled subnets is alleviated in this work by making the subnets leaky and in the process of doing so, virtually breaking the hierarchy. The utilization of leaky-walled subnets as opposed to hard-walled subnets reduces the congestion in accessing the top level network. The proposed approach does not alter the layout of the hierarchical NoCs except for the additional links to establish the leaky walls. There are no changes in the router design as the leaks are made available through merely activating the unused ports of the existing routers on the periphery of the hard-walled subnets as shown in Figure 2 and discussed in detail in Section 4. In general, the proposed leaky-walled approach can be applied to any hierarchical NoC regardless of the topology of the hierarchical layers. In this article, its application is primarily discussed for the hierarchical mesh-mesh NoC shown in Figure 2(a) for ease of demonstration.

(II) Employing a locality-aware network utilization balancing technique for NoCs with broken hierarchy and multiple networks. The resulting network topology with the broken network hierarchy is essentially a composition of two different network types serving each core-the bottom level network and the top level network. The injection of packets into the top level or bottom level network is selected based on the locality of the destination node as opposed to the selection based solely on the subnet membership of the destination node-the leaky-walled approach. The application of the proposed locality-aware packet injection (quantified by the network utilization ratio defined in this work) balances the number of packets injected into each of the networks. Through the application of the proposed locality-aware network utilization balancing technique, the same amount of resources (area and power) of the NoC are utilized more efficiently, as empirically proven by the synthetic exascale CMP traffic loads used in the literature.

In the literature, there exist $\mathrm{NoC}$ topologies with multiple networks, similar to the networks resulting from breaking the hierarchy in traditional hierarchical NoCs to form multiple networks for the same CMP. In general, if a network architecture is comprised of multiple networks (homogeneous as in the case of meshX2 (Figure 1(c)) 
and CMeshX2 (Figure 1(d)) NoCs or heterogeneous as in the case of a NoC with the broken hierarchy), the utilization of the various networks must be balanced. As such, the network utilization balancing technique proposed in this work is the second major novelty in this article, shown to be necessary not only for networks whose hierarchy is broken but also for existing multi-network NoCs in the literature. The hardware overhead required for the implementation of the proposed network utilization balancing technique is discussed in Section 4 and Section 5 for the hierarchy-broken and multi-network NoCs, respectively.

\subsection{Small-World-Like Properties of Hierarchical and Multi-Network NoCs}

The networks evaluated in this work feature long-range links and clustering of nodes. The presence of such features are characteristic of small-world networks [Ogras and Marculescu 2006]. Specifically, the long-range links introduce short internode distances $(\mu)$, and clustering of nodes provides a high degree of clustering (clustering coefficient $C$ ). This work evaluates the hierarchical and multi-network NoC topologies for small-world-like properties. However, as shown in this work, the congestion phenomenon is the defining factor for the performance of the hierarchical networks. The traditional small-world properties of average internode distance $(\mu)$ and clustering coefficient $(C)$ do not take into account the congestion phenomenon, thereby failing to accurately predict the performance of such networks. This work shows the necessity of simulation-based analysis of such complex networks by highlighting the difference in prediction of network performance between the theoretical analysis of small-world-like properties (Section 6) and simulation-based analysis (Section 7). The third novelty of this work is the analytical analysis of the small-world like properties of average internode distance $(\mu)$ and clustering coefficient $(C)$ of the complex networks.

As shown empirically here, the necessity of the application of a network utilization technique, such as proposed in this work is more critical in larger networks. This is analogous to the elevator system of a skyscraper consisting of multiple carriages (analogous to a NoC with multiple networks) [Strang and Bauer 2007]. In the elevator system, the throughput can be increased simply by increasing the number of carriages. Similarly, in an NoC architecture, the throughput can be increased by increasing the number of networks (like the meshX2 and CMeshX2 NoC topologies). However, simply increasing the number of carriages (or number of networks) is not efficient. In order to achieve throughput efficiency and energy efficiency, the elevator systems balance the traffic by dedicating certain carriages to certain floors-zoning of floors. This balancing of load becomes more critical as the height of the building increases [Strang and Bauer 2007]. Similarly, in NoC architectures consisting of multiple networks, network utilization balancing is crucial and its criticality is further exacerbated for large networks (like the height of the skyscraper). The application of the proposed network utilization balancing technique provides a substantial increase in the network performance and cost efficiency for CMPs consisting of high hundreds to thousand cores.

The rest of the article is structured as follows. The existing body of work in relation to this effort is presented in Section 2. The motivation for breaking the hierarchy in the hierarchical mesh-mesh $\mathrm{NoC}$ is presented in Section 3. The NoC architecture that breaks the hierarchy and the locality-aware network utilization balancing technique are presented in Section 4. The extension of the locality-aware network utilization balancing technique, developed for the NoC with the broken hierarchy, is extended to multiple networks in Section 5. The theoretical analysis of the small-world like properties of the networks evaluated in this work is presented in Section 6. The evaluation methodology is presented in Section 7. The results and summary are presented in Section 8 and Section 9, respectively. 


\section{BACKGROUND}

The NoC topology is a critical factor in determining the network performance (throughput and energy consumption) and hence the efficiency of exascale CMPs. Since the flat-mesh NoC does not scale efficiently with the increase in the network diameter, several high radix and hierarchical topologies have been proposed. High radix topologies such as flattened butterfly [Kim et al. 2007], fat-tree and tapered fat-tree [Balfour and Dally 2006] reduce the average hop count in the network but result in higher router complexity, energy consumption, and area utilization per router. The use of long-range links to reduce the average hop count is also proposed in Ogras and Marculescu [2006] and Wang et al. [2003].

The scalability challenges posed by the flat-mesh 2D-NoC are proposed to be tackled by hierarchical NoCs as in Balfour and Dally [2006], Bourduas and Zilic [2007], and Das et al. [2009]. A concentrated mesh (CMesh) NoC, as depicted in Figure 1(a), is proposed in Balfour and Dally [2006], where multiple cores are attached to the same router, as opposed to a single core per router in the flat-mesh, so as to decrease the network diameter. In Bourduas and Zilic [2007], a hybrid ring-mesh NoC, as depicted in Figure 1(e), is proposed, wherein a larger mesh-network is broken up into smaller submeshes and the submeshes in turn are connected using hierarchical rings. In Das et al. [2009], a similar hybrid NoC is proposed, where the cores are broken up into smaller clusters interconnected using a local bus. Each of the buses are then interconnected in a mesh structure, as depicted in Figure 1(g). The hierarchical NoC structures proposed in Balfour and Dally [2006], Bourduas and Zilic [2007], and Das et al. [2009] utilize the hard-walled subnets, as discussed in Section 1. In this work, a qualitative (Section 3) and a quantitative (Section 8) discussion is provided to highlight the bottleneck problem in such hard-walled hierarchical NoCs and a low overhead solution is provided to improve the efficiency of the traditional hierarchical NoCs.

The performance benefits of using multiple networks such as meshX2 [Figure 1(c)] and CMeshX2 [Figure 1(c)], in which two similar networks service each core, are shown in Balfour and Dally [2006]. Further, the performance trade-offs involved in using multiple networks versus a single network with more virtual channels (VCs), is discussed in Yoon et al. [2010]. Under the assumption that the wire area and energy requirements are less than those of buffers, it is concluded in Yoon et al. [2010], that utilizing multiple networks is more energy efficient but at a loss of performance as compared to a single network with more VCs. However, since the VCs essentially time multiplex the physical links to provide improved throughput, their scalability is limited after a certain number of VCs (due to lack of empty time slots on the physical channel). In this work, a network utilization balancing technique is proposed to increase the throughput of the multiple networks without compromising energy efficiency.

\section{MOTIVATION FOR BREAKING THE HIERARCHY}

Recent work on network traffic characterization in Soteriou et al. [2006], Bezerra et al. [2010], and Heirman et al. [2008] have investigated the localization of traffic on NoCs. However, it is shown in Manevich et al. [2012], that while this is true for small network sizes, for large network sizes (which will be typical of exascale computing CMPs) the global traffic is the bottleneck to the entire NoC (blocking the local traffic as well) and consequently the CMP. Hence, it is necessary to efficiently handle the global traffic in large NoCs for future CMPs. Furthermore, the exascale computing study in Kogge et al. [2008] reports that the scientific workloads for the exascale computing CMPs are expected to be nonlocalized. Given such requirements for the global traffic, there is a need for an efficient $\mathrm{NoC}$ architecture that can sustain high throughput local communication and can also scale efficiently in global communication. 
Each of the hierarchical NoCs discussed in Balfour and Dally [2006], Bourduas and Zilic [2007], and Das et al. [2009] are capable of efficiently reducing the average hop count between the cores, resulting in a low zero-load latency. However, in all the hierarchical NoCs, if a core inside a subnet needs to communicate with a core inside another subnet (inter subnet traffic), it has to access the top level network through a single access point. These traditional hierarchical NoCs do not provide links at the cores in the bottom level network to connect to a core outside the subnet. Such a hard-walled partitioning of the network is very inefficient, as it requires cores in the neighboring subnets placed physically next to each other (due to the planar structure of the 2DICs) to communicate through the top level network, thereby, increasing the hop count and adding to the congestion. This congestion phenomenon is not highly significant in smaller NoCs (on the order of 100 cores) since the amount of inter subnet traffic is low. However, in larger NoCs targeted for exascale computing CMPs, the bottleneck in acquiring the top level network is the major contributing factor in the reduced throughput. The hierarchical NoC topologies exploit traffic localization by providing high bandwidth connections to the cores in the same subnet, but are highly inefficient in handling global traffic. The efficiency in serving the global traffic is the determining factor for the communication energy and performance efficiency (especially for large network sizes), as observed in Das et al. [2009] and Manevich et al. [2012].

This work utilizes the proposed locality-aware network utilization balancing technique in order to provide both throughput and energy efficient communication for local as well as global traffic with minimal design complexity. This work also utilizes existing router designs and routing algorithms. Consequently, the focus is on how to efficiently use the existing NoC designs for future large CMPs, postulated by theoretical discussions and empirical verifications with synthetic exascale traffic loads (given the lack of real workloads for exascale study and precedence of synthetic loads in the literature).

\section{NETWORK ARCHITECTURE FOR BREAKING THE HIERARCHY}

The design scheme used to break the hierarchy in hierarchical NoCs is presented in Section 4.1. The locality-aware strategy employed to select the top level network vs. the bottom level network and the methodology used to balance the packet injection between the two networks are discussed in Section 4.2. The effect of the network utilization ratio on the NoC performance is discussed quantitatively in Section 4.3.

\subsection{Network Structure}

The network architecture used to break the hierarchy (depicted in Figure 2(b)) partitions the cores into subnets. In general, the subnets can be of any size and different subnets can be of different sizes. Without loss of generality, for the purposes of evaluation in this work, the subnets are of equal size, as depicted in Figure 2(b). If the subnets are of different sizes, a similar technique can still be employed for breaking the hierarchy (the network utilization balancing strategy is discussed in Section 4.2). As an example for breaking the hierarchy, consider a hierarchical mesh-mesh NoC. In a hierarchical mesh-mesh NoC, each core inside the subnet is connected to a router and the routers are interconnected using a mesh topology, forming the bottom level network. In addition, each subnet also consists of a hub and each of the cores are connected to the central hub. The hubs are interconnected at the top level in a mesh forming the top level network. In this work, in addition to the access point to the top level network, the cores at the boundaries of the subnets are connected to cores at the boundary of the adjacent subnets like a regular flat-mesh-leaky-walled subnets-thereby virtually breaking the hierarchy, as depicted in Figure 2(b). The resulting NoC topology essentially consists of two different networks serving each core, the flat-mesh at the bottom level and the CMesh at the top level-a heterogeneous multi-network NoC (as 
opposed to homogeneous multi-network NoCs in the case of meshX2 and CMeshX2). Using a leaky-walled approach to the interconnection of subnets eases the bottleneck in acquiring the top level network, thereby increasing the NoC throughput, while still providing low latency communication compared to the flat-mesh NoC due to its hierarchical structure. Although, in this work, a hierarchical mesh-mesh NoC topology, as depicted in Figure 1(f) is used for ease of demonstration a similar technique of interconnecting subnets can be employed as long as the overhead of doing so is low. For example, in a mesh-ring hierarchical NoC, as depicted in Figure 1(e), a similar technique of interconnecting the subnets can be employed to virtually break the hierarchical NoC into a flat-mesh NoC at the bottom level and a ring-based NoC at the top level.

\subsection{Locality-Aware Packet Injection for Balancing Network Utilization}

In a traditional hierarchical $\mathrm{NoC}$, the injection of the packets into the top level network or the subnet network depends solely on the subnet membership of the destination. Instead, in the proposed network design scheme, the locality of the packet destinations in relation to the packet source (not just the subnet membership of the destination node) impacts the injection of the packet to the top or bottom level network. The objective is to balance the number of packets injected by each core between the top and bottom level networks. If the number of packets injected into the top level network is high compared to the bottom level network, then the proposed NoC topology will suffer from the same bottleneck in accessing the top level network as experienced by the hardwalled NoC topologies. Instead, if the number of packets injected into the bottom level network is high compared to the top level network then the NoC topology will suffer from the scalability problems of the bottom level network.

The network packet injection is balanced using the local router range parameter, defined as the distance from the destination node within which the source node injects packets into the bottom level network instead of the top level network. The following scenarios occur.

(S1) If the destination core is in the same subnet as the source core, then the packet is to be routed on the bottom level (flat-mesh) network.

(S2) If the destination core is not in the same subnet as the source core, then the packet is still routed on the bottom level (flat-mesh) network if the number of hops between the source and destination cores is less than a certain local router range. This scenario utilizes the leaky-walled property of the proposed network topology and is unlike the hard-walled hierarchical network topologies.

(S3) If the destination and source cores are not in the same subnet and the number of hops between them is greater than the local router range, then the packet is to be routed on the top level (CMesh) network.

The selection of the network for packet injection is done deterministically at the network interface, thereby keeping the design overhead minimal by not requiring any change in the router designs of either of the multi-network NoCs. Once the network interface at the source determines the network into which the packet is to be injected (depending on the three scenarios), the packet is routed on that particular network until it reaches its destination. Since the application of the proposed locality-aware network balancing technique does not require the packet to be routed back and forth between the networks in the multi-network NoC, no change is required in the routing protocol of either of the networks. As such, this technique of network utilization balancing can be applied to any multi-network NoC topology that has a definition for distance between cores. As an example, in this work the technique is applied to multi-network NoC topologies, where the distance is defined as the Manhattan-distance between cores. 
If the local router range is set to 0 then scenarios S1 and S2 are combined and the subnet partitioning becomes similar to the hierarchical hard-walled partitioning, and the number of packets injected into the top level network is higher than that of the bottom level network. As the value of the local router range is increased, the subnets become more leaky-walled (some of the destination nodes move from scenario S3 to scenario S2), thereby transferring some of the packets from the top level network to the bottom level network. Quantitatively, the distribution of the packets between the top and bottom level networks is defined by the network utilization ratio, netUR. The network utilization ratio is defined in this work, as the ratio of the total number of destination cores to which the packets are routed on the top level network over the total number of destination cores to which the packets are routed on the bottom level:

$$
n e t U R=\frac{P_{S 3} N_{S 3}}{P_{S 1} N_{S 1}+P_{S 2} N_{S 2}},
$$

where $N_{S 1}$ is the total number of destination cores in each subnet (scenario S1), $N_{S 2}$ is the total number of destination cores outside the subnet but in the local router range utilizing the bottom level network (scenario S2), $N_{S 3}$ is the total number of destination cores outside the subnets and outside the local router range (scenario S3) and $P_{S i}$ is the fraction of the total number of packets sent to each core in each of the three scenarios.

For uniform random traffic, the fraction of the total number of packets sent to each core in the network is equal and Equation (1) reduces to

$$
\text { net } U R_{\text {uniform }}=\frac{N_{S 3}}{N_{S 1}+N_{S 2}} \text {. }
$$

Similarly, for a subnet localized traffic pattern, where a fraction $p_{\text {sub }}$ of the packetsthe localization degree-are distributed uniformly to the cores inside the subnet, while the remaining $1-p_{s u b}$ fraction of the packets are distributed uniformly to the cores outside the subnet, the network utilization ratio, net $R_{\text {localized }}$ is formulated as

$$
n e t U R_{\text {localized }}=\frac{\frac{1-p_{\text {sub }}}{N_{\text {total }}-N_{\text {sub }}} N_{S 3}}{\frac{p_{\text {sub }}}{N_{\text {sub }}} N_{S 1}+\frac{1-p_{\text {sub }}}{N_{\text {total }}-N_{\text {sub }}} N_{S 2}},
$$

where $N_{\text {sub }}$ and $N_{\text {total }}$ are the number of cores in a subnet and the total number of cores in the network, respectively.

Increasing the local router range moves the destination cores from scenario $\mathrm{S} 3$ to scenario S2 (the value of $N_{S 2}$ increases while the value of $N_{S 3}$ decreases), thereby decreasing the netUR as governed by Equation (1). The effects of the local router range and consequently the netUR on the network performance are discussed next.

\subsection{Effect of Network Utilization Ratio on Performance}

The effects of varying the local router range (and consequently the netUR) on the (1) latency, (2) throughput, and (3) energy/flit for a 1024-core CMP under uniform traffic are shown in Figure 3. For a local router range equal to 0 (the traditional, hierarchical mesh-mesh topology), corresponding to a very high netUR $(=340)$ (square markers in Figure 3), the subnet partitioning becomes hard-walled. Under such a scenario, the performance of the NoC with the broken hierarchy (combination of flatmesh and CMesh) becomes similar to that of the CMesh alone (diamond markers). This shows the bottleneck due to the hard-walled subnets in the traditional hierarchical NoC topologies. Conversely, for a high local router range $(=48)$, corresponding to a very low netUR $(=0.01)$ (star markers), the network performance of the NoC with the broken hierarchy (combination of the flat-mesh and CMesh) becomes similar to that of the flatmesh alone (rightwards pointing triangle markers). This is because the majority of the 


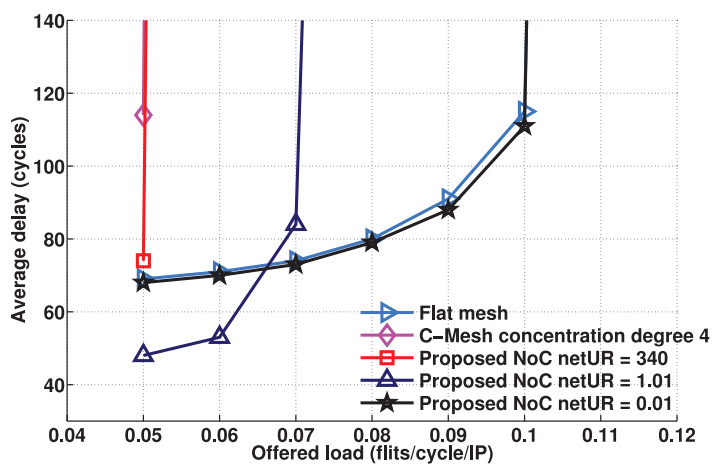

(a) Latency.

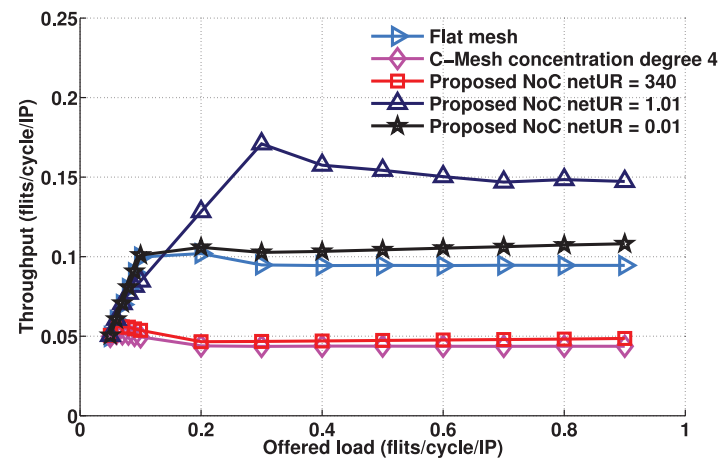

(b) Throughput

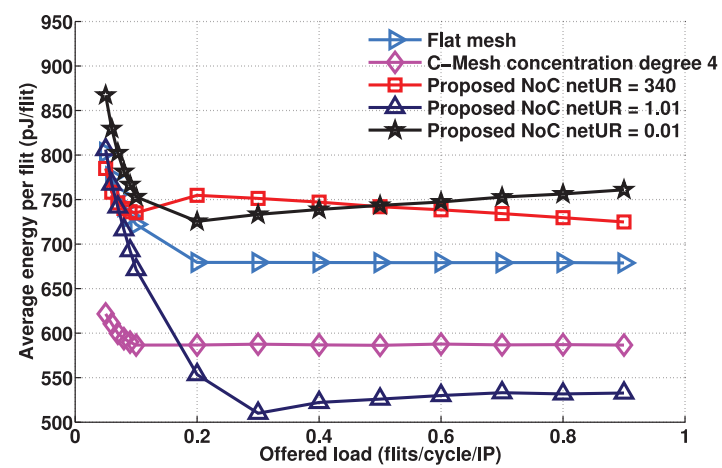

(c) Energy per flit.

Fig. 3. Effect of network utilization ratio netUR on the performance of a 1024-coresNoC.

packets are now being routed on the bottom level mesh network. Therefore, to balance the number of packets injected into the top and bottom level networks equally-while breaking the hierarchy-a netUR approximately equal to 1 (upwards pointing triangle markers) is chosen in this work. For all the cases analyzed in this work, a netUR closest to 1 provides for the best performance compared to all other network utilization ratios. A similar trend is observed for the other network sizes of 512, 256, and 100 cores (not shown), evaluated in this work. In the experimentation, similar trends are seen at all network sizes. However, for small NoCs the effects are not as prominent. 
The choice of the local router range is solely based on balancing the number of packets injected into each of the top or bottom level networks. It is emphasized that this selection is contrary to the selection based on the number of hops between the source and destination nodes. If the choice of the local router range would be based on the number of hops between the source and the destination nodes, then the maximum value of the local router range, irrespective of the traffic distribution, would be 1 . This is because except for communication between the cores at the boundaries of the subnet, the shortest path is through the top level network. In such an undesired case, the proposed NoC with the broken hierarchy (combination of flat-mesh and CMesh) would suffer from the same bottleneck problems as the hard-walled hierarchical NoCs, thereby resulting in a reduced throughput. The underutilization of the bottom level (flat-mesh) network increases its static energy consumption as discussed in Soteriou et al. [2006], resulting in a higher overall energy/flit for the entire network. This reduced throughput and higher energy consumption results in a lower network efficiency. Hence a local router range should be selected using (1), such that it balances the top and bottom network utilization.

Therefore, to summarize, the minimal changes required in a traditional hierarchical NoC topology (1) to break the hierarchy and (2) to implement the proposed localityaware network utilization balancing technique discussed in Section 4.2, are:

(1) additional links to connect unused ports of routers at subnet boundaries;

(2) register(s) in the network interface to program the local router range, and

(3) a circuit in each network interface to calculate and compare whether the distance of the destination node from the source node is less than or equal to the local router range.

Since only the unused ports of routers at the subnet boundaries need to be connected, only a limited and finite number of additional links are required to break the hierarchy-keeping the overhead low. For instance, a router on the East boundary of a subnet only needs additional links at the East port to connect to the West port of the router on the West boundary of the adjacent subnet. The energy and area overheads of these additional links have been accounted for in the simulation-based analysis performed in this work. Furthermore, the calculation and comparison of the distance between the source and destination nodes can be done using simple ALU circuits. Therefore, the additional hardware is lightweight and the proposed solution is highly practical. The minimal changes proposed in this work improve the energy- and performance-efficiency of the NoC by making efficient use of the existing resources in the network.

\section{EXTENSION OF NETWORK UTILIZATION BALANCING TO MULTI-NETWORK NOCS}

Breaking the hierarchy of a hierarchical NoC results in two different networks serving each core. Given the similarity in employing two separate networks, the locality-aware network utilization balancing technique discussed in Section 4.2 can be directly extended to multi-network NoC architectures. In this work the network utilization balancing technique is employed for the meshX2 and CMeshX2 NoC topologies depicted in Figure 1. The key strategy employed is the separation of local and global communication and reserving each of the multiple networks for each of the communication types. This is analogous to the strategy employed for separation of elevator traffic in sky-scrappers as discussed in Section 1 [Strang and Bauer 2007].

In the case of the CMeshX2 NoC, the number of cores in the subnet (Scenario S1) is the concentration degree of the CMesh topology. In the case of meshX2 NoC, the number of cores in Scenario S1 is equal to the number of neighboring cores in the hop-radius equal to the size of the subnet. The local router range which consequently defines 
the network utilization ratio depends on the traffic type (defined by Equation (2) and Equation (3) for a uniform traffic and localized traffic pattern, respectively). In general, the local router range should be selected based on the expected traffic patterns of the CMP. A higher degree of localization of the traffic results in a lower local router range for the same network utilization ratio and vice-versa.

Similar to the case of the hierarchy broken NoCs, the application of the network utilization balancing technique to multi-network NoCs is lightweight and practical in terms of its additional hardware requirements. Unlike the hierarchy broken NoCs, the application of the network utilization balancing technique to multi-network NoCs does not require any additional links to break the hierarchy to result in multiple networks. Since the multi-network NoCs already consist of multiple networks, the changes required are only in the network interface. The additional hardware requirements at the network interface are:

(1) register(s) to program the local router range; and

(2) a circuit to calculate and compare whether the distance of the destination node from the source node is less than or equal to the local router range.

These changes required at the network interface for multi-network NoCs are the same as the changes required at the network interface for hierarchy broken NoCs. As in the case of the hierarchy broken NoCs, the calculation of the distance between the source and destination nodes and its comparison to the local router range can be done using simple and lightweight ALU circuits.

\section{SMALL-WORLD-LIKE PROPERTIES OF EVALUATED NETWORKS}

As discussed in Section 1.2, the networks evaluated in this work have small-worldlike features of long range links and clustering. This section provides a quantitative discussion of the small-world like features of average inter-node distance and clustering coefficient in Section 6.1 and Section 6.2, respectively.

\subsection{Average Inter-Node Distance}

The average inter-node distance is a measure of the average number of hops required in the network for a given traffic pattern. It is desirable to have a lower average inter-node distance since it results in lower latency and lower energy consumption. The average inter-node distance $\mu$ is computed using (4):

$$
\mu=\sum_{i} \sum_{j \neq i} f_{i j} d(i, j) \quad \forall i, j \in P,
$$

where, $P$ is the set of processing elements $(\mathrm{PE})$ in the network, $f_{i j}$ is the frequency of communication between node $i$ and node $j$, and $d(i, j)$ is the distance between nodes $i$ and $j$ [Ogras and Marculescu 2006]. In general, the communication frequency $f_{i j}$ between nodes $i$ and $j$ is obtained using (5):

$$
f_{i j}=\frac{V_{i j}}{\sum_{p} \sum_{p \neq q} V_{p q}} \quad \forall i, j, p, q \in P,
$$

where $V_{i j}$ is the communication volume between nodes $i$ and $j$.

The distance $d(i, j)$ between nodes is a function of the NoC topology. For a regular mesh structure, $d_{M}(i, j)$ is computed as the Manhattan distance between the processing elements (PEs) $i$ and $j$, whereas for a CMesh network, $d_{C M}(i, j)$ is computed as the Manhattan distance between the routers to which the PEs $i$ and $j$ belong, that is,

$$
d_{M}(i, j)=\left|i_{x}-j_{x}\right|+\left|i_{y}-j_{y}\right|
$$




$$
d_{C M}(i, j)=d\left(r_{i}, r_{j}\right)=\left|r_{i_{x}}-r_{j_{x}}\right|+\left|r_{i_{y}}-r_{j_{y}}\right|,
$$

where, $r_{i}$ and $r_{j}$ are the routers to which PEs $i$ and $j$ belong respectively, and the subscripts $x$ and $y$ are the $\mathrm{x}$ - and y-coordinates, respectively. For the traditional hierarchical mesh-mesh NoC and the NoC with a broken hierarchy, the distance between PEs depends on the network in which the packets are routed. Hence, the distance $d(i, j)$ between PEs $i$ and $j$ is defined as

$$
d(i, j)= \begin{cases}d_{M}(i, j), & \text { if routed on bottom layer } \\ d_{C M}(i, j), & \text { if routed on top layer }\end{cases}
$$

Note that, though the discussion in this section pertains to the mesh, CMesh and hierarchical mesh-mesh topologies, the definition of the average inter-node distance can be extended to any topology that has a definition of distance. For instance, in the mesh-ring topology the distance $d(i, j)$ is equal to the Manhattan-distance between PEs if the packets are routed on the bottom layer mesh network or the number of hops on the ring if the packets are routed on the top layer ring network.

\subsection{Clustering Coefficient}

The clustering coefficient is a measure of how tightly the nodes are interconnected in a network [Ogras and Marculescu 2006]. A higher clustering coefficient implies more direct links between nodes, thereby reducing the energy consumption. Traditionally, the clustering coefficient $C_{i}$ of a node $i$ is defined as the ratio of the number of links $l_{i}$ existing in the network between its neighbors $n_{i}$ to the maximum possible number of links [Ogras and Marculescu 2006], that is,

$$
C_{i}=\frac{2 l_{i}}{n_{i}\left(n_{i}-1\right)}
$$

However, the definition of clustering coefficient in (9) does not accurately take into account the heterogeneity of interaction between nodes in complex networks [Saramäki et al. 2007], such as the ones discussed in this work. As such, an alternative weighted definition of the clustering coefficient defined in Barrat et al. [2004], which takes into account the heterogeneity in interaction between nodes is utilized in this work. The weighted clustering coefficient is given as

$$
\widetilde{C}_{i}=\frac{1}{n_{i}\left(n_{i}-1\right)} \sum_{j, k} \frac{1}{\left\langle w_{i}\right\rangle} \frac{w_{i j}+w_{i k}}{2} a_{i j} a_{j k} a_{i k},
$$

where $n_{i}$ is the number of neighbors of the node $i$. The term $w_{i j}$ is the weight of the link between nodes $i$ and $j$. The term $w_{i}$ is the average value of the weights of links to node $i$, that is, $\left\langle w_{i}\right\rangle=\sum_{j}\left(w_{i j} / n_{i}\right)$. The term $a_{i j}=1$ if there is a link between nodes $i$ and $j$ and $a_{i j}=0$ otherwise. The clustering coefficient of the entire network $C_{N}$ is obtained by taking the average of the clustering coefficients $C_{i}$ of each of the nodes, that is, for a network with a set of PEs $P$ with $N_{p}$ number of nodes, $C_{N}$ is given by

$$
C_{N}=\frac{1}{N_{p}} \sum_{i \in P} C_{i}
$$

The nodes in the networks evaluated in this work interact with each other either through a single router (e.g. nodes connected to the same router in a CMesh) or through two routers (e.g. nodes in adjacent subnets or adjacent nodes in a mesh network). In this work, a link is defined as a connection between two nodes that consists of two or less routers. Similarly, a node $j$ is a neighbor of node $i$ if they are connected to each 
Table I. Configuration of Evaluated NoC Topologies

\begin{tabular}{|c|c|c|c|}
\hline Number of cores & Flat-mesh $\backslash$ meshX2 & CMesh $\backslash$ CMeshX2 & Hierarchical mesh-mesh \\
\hline 100 & $10 \times 10$ mesh & $\begin{array}{c}5 \times 5 \text { mesh } \\
\text { concentration degree }=4\end{array}$ & $\begin{array}{r}\text { top level mesh }=5 \times 5 \\
\text { sub-net } \text { mesh }=2 \times 2\end{array}$ \\
\hline 256 & $16 \times 16$ mesh & $\begin{array}{c}8 \times 8 \text { mesh } \\
\text { concentration degree }=4\end{array}$ & $\begin{array}{r}\text { top level } \text { mesh }=8 \times 8 \\
\text { sub-net } \text { mesh }=2 \times 2\end{array}$ \\
\hline 512 & $16 \times 32$ mesh & $\begin{array}{c}8 \times 16 \text { mesh } \\
\text { concentration degree }=4\end{array}$ & $\begin{array}{c}\text { top level } \text { mesh }=8 \times 16 \\
\text { sub-net } \text { mesh }=2 \times 2\end{array}$ \\
\hline 1024 & $32 \times 32$ mesh & $\begin{array}{c}16 \times 16 \text { mesh } \\
\text { concentration degree }=4\end{array}$ & $\begin{array}{c}\text { top level mesh }=16 \times 16 \\
\text { sub-net } \text { mesh }=2 \times 2\end{array}$ \\
\hline
\end{tabular}

Table II. Small-World-Like Properties of Evaluated NoC Topologies

\begin{tabular}{|c|c|c|c|c|c|}
\hline $\begin{array}{c}\text { Number } \\
\text { of cores }\end{array}$ & Metric & $\begin{array}{c}\text { Flat-mesh } \backslash \\
\text { meshX2 }\end{array}$ & $\begin{array}{c}\text { CMesh } \backslash \\
\text { CMeshX2 }\end{array}$ & $\begin{array}{c}\text { Hierarchical } \\
\text { mesh-mesh }\end{array}$ & $\begin{array}{c}\text { Hierarchical mesh-mesh } \\
\text { with broken hierarchy }\end{array}$ \\
\hline \multirow{3}{*}{100} & $C_{N}$ & 0 & 0.303 & 0.322 & 0.314 \\
\cline { 2 - 6 } & $\mu$ & 6.667 & 3.232 & 3.273 & 4.287 \\
\cline { 2 - 6 } & $C_{N} / \mu$ & 0 & 0.094 & 0.098 & 0.073 \\
\hline \multirow{3}{*}{256} & $C_{N}$ & 0 & 0.285 & 0.304 & 0.296 \\
\cline { 2 - 6 } & $\mu$ & 10.667 & 5.271 & 5.286 & 6.956 \\
\cline { 2 - 6 } & $C_{N} / \mu$ & 0 & 0.054 & 0.056 & 0.043 \\
\hline \multirow{3}{*}{512} & $C_{N}$ & 0 & 0.278 & 0.297 & 0.289 \\
\cline { 2 - 6 } & $\mu$ & 16 & 7.953 & 7.961 & 10.080 \\
\cline { 2 - 6 } & $C_{N} / \mu$ & 0 & 0.035 & 0.037 & 0.029 \\
\hline \multirow{3}{*}{1024} & $C_{N}$ & 0 & 0.271 & 0.291 & 0.282 \\
\cline { 2 - 6 } & $\mu$ & 21.333 & 10.635 & 10.639 & 13.791 \\
\cline { 2 - 6 } & $C_{N} / \mu$ & 0 & 0.025 & 0.027 & 0.020 \\
\hline
\end{tabular}

other through two or less routers. Furthermore, if a link consists of a single router, then the link is assigned a weight $w_{i j}=1$, and if a link consists of two routers it is assigned a weight $w_{i j}=1 / 2$. Using these definitions of the neighbors, link and the corresponding weights the clustering coefficient of each of the networks can be calculated as shown in Appendix A.

The small-world-like properties of average inter-node distance $(\mu)$ and clustering coefficient $(C)$ of the networks evaluated in this work configured as shown in Table I are shown in Table II. The theoretical analysis using the small-world properties of $\mu$ and $C$ suggests that the traditional hierarchical mesh-mesh structure will provide the best performance (highest $C_{N} / \mu$ in Table II). For instance, for 1024 cores, theoretical analysis with small-worldness suggests that the $C / \mu$ metric of small-world like property is $35 \%$ higher for a hierarchical mesh-mesh $(C / \mu=0.027)$ compared to the hierarchy-broken one $(C / \mu=0.020)$. The data in Table II also suggests that there is no benefit to be obtained from breaking the hierarchy as indicated by the increased average internode distance $\mu$. For instance, for 1024 cores, theoretical analysis with small-worldness suggests that the average internode distance is $23 \%$ lower for a hierarchical mesh-mesh $(\mu=10.639)$ compared to the hierarchy-broken hierarchical mesh-mesh NoC ( $\mu=13.791)$. However, as discussed qualitatively in Section 1.2, the static theoretical analysis of small-world-like properties does not take into account the congestion phenomenon. As such, the theoretical analysis using small-world-like properties falls short in identifying the substantial benefits of network utilization balancing. This is expected because network utilization balancing targets precisely this congestion bottleneck in complex hierarchical and multi-network systems. Simulation-based analysis, which accounts for congestion on the other hand, shows that the performance 
of the NoC is improved by using the proposed locality-aware network utilization balancing technique on multi-network NoCs (homogeneous like meshX2 and CMeshX2 or heterogeneous like hierarchical NoC with broken hierarchy). The results of the simulation-based analysis being counter-intuitive to those generated in the theoretical analysis reveal the deficiency of small-world metrics in capturing the performance of targeted multi-network NoCs, and assert the importance of the simulation-based analysis presented in this work.

\section{SIMULATION BASED EVALUATION METHODOLOGY}

All combinations of the discussed network and packet injection types are analyzed in order to comprehensively observe the impact of the network topology and localityaware packet injection on the performance of many-core CMPs. In particular, the following network types are evaluated and compared in this work: (1) the flat-mesh NoC, (2) the meshX2 NoC with random network selection for packet injection, (3) the meshX2 NoC with the proposed locality-aware network utilization (NU) balancing technique for packet injection, (4) the CMesh NoC, (5) the CMeshX2 NoC with random network selection for packet injection, (6) the CMeshX2 NoC employing the locality-aware network utilization (NU) balancing for packet injection, (7) the traditional hard-walled mesh-mesh NoC, (8) the NoC with broken hierarchy (combination of flat-mesh and CMesh) employing the locality-aware network utilization (NU) balancing for packet injection. The network types are evaluated using a cycle-accurate NoC simulator built using SystemC. The flat-mesh and CMesh NoCs are traditional, with a single network serving each core, while meshX2 and CMeshX2 with two networks serving each core and the traditional hard-walled mesh-mesh NoCs have the same hardware overhead (normalized in terms of the area of the routers and links) as the NoC with broken hierarchy (combination of flat-mesh and CMesh). The NoC topologies are evaluated and compared for 100, 256, 512, and 1024-core CMPs.

In general, each of the individual networks in a multi-network NoC can use its own routing algorithm. For ease of discussion of theory and results, all the networks evaluated in this work utilize a worm-hole-credit-based flow control dimension order XY routing algorithm. The routers in the flat-mesh $\backslash$ meshX2 NoCs and the bottom level of the hierarchical NoC utilize four virtual channels (VC) with an arbitrary buffer depth of two flits each. While the routers in the CMesh $\backslash$ CMeshX2 NoCs and the hubs in the subnets of the top level network of the hierarchical NoC utilize four VCs with a buffer depth of five flits each (to ensure the number of buffers at each router is the same as that of the flat-mesh-based topologies for a cluster of four cores (the size of the subnet)). The packet size is fixed at 10 flits of 32-bits each. While the selection of buffer sizes is simplistic and arbitrary, it is observed (not reported for brevity) that the following presented trends are preserved for other sizes, as well.

Since the current benchmark parallel applications do not scale for large CMPs (hundreds of cores), the NoCs are evaluated using synthetic traffic patterns. In this work, the network performance is evaluated using uniform traffic as well as localized traffic to emulate both the localized and non-localized application cases. The subnet localized traffic pattern is evaluated with localization degrees of $0.25,0.50$, and 0.75 representing a balanced and low (expected by Kogge et al. [2008]), and a high (expected based on current workloads) rate of communication between neighboring cores in a CMP. The equivalent of the subnet localized traffic for a flat-mesh is the concentration of the traffic to the neighboring cores in the hop-radius equal to the size of the subnet. The structure of the evaluated NoCs is shown in Table I. To keep the evaluation consistent, the concentration degree (number of cores served by a single router) of the CMesh $\backslash$ CMeshX2 and the number of cores in the subnet mesh of the proposed NoC and the traditional hierarchical mesh-mesh are kept the same. 


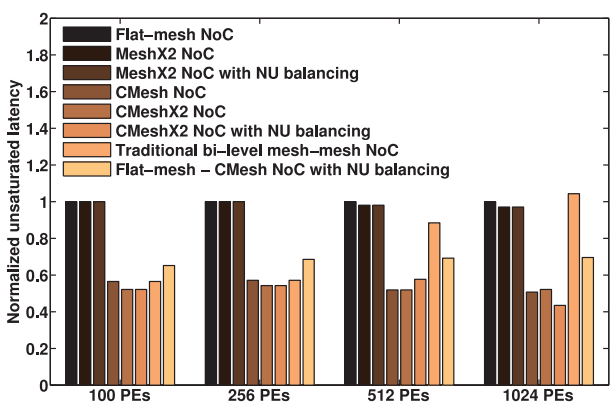

(a) Unsaturated latency.

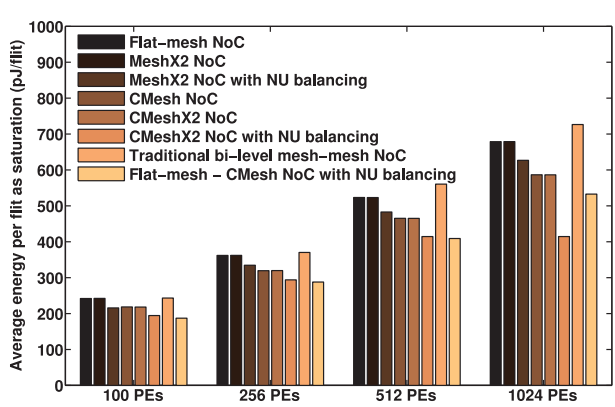

(c) Energy/flit at saturation.

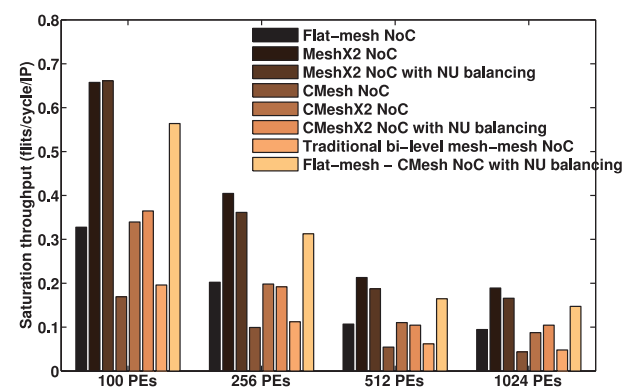

(b) Saturated throughput.

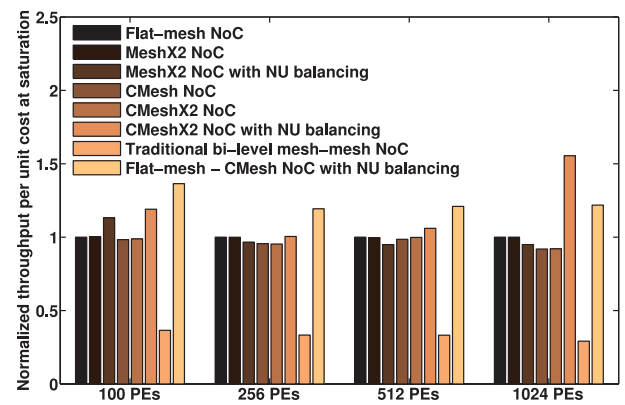

(d) Normalized throughput/cost at saturation.

Fig. 4. Network performance comparison for a uniform random traffic pattern.

The NoCs are analyzed for throughput, energy consumption, and area utilization. The energy efficiency of the NoCs is evaluated using the average energy/flit, while the throughput efficiency is evaluated using the throughput per unit cost, where the cost is the energy-area product. The energy and area consumption of the router and the wired links are modeled using the Orion [Kahng et al. 2009] power and area models. It is argued that the network that can sustain a high throughput local communication and scale efficiently for global communication, should show superior performance efficiency under both the uniform and localized traffic cases analyzed in this work.

\section{NETWORK ANALYSIS RESULTS}

The NoC architectures are evaluated for latency (Section 8.1) as well as throughput and energy efficiency (Section 8.2), as outlined in Section 7. Next, the proposed energy- and performance-efficient scaling of the NoCs are demonstrated in Section 8.3. Finally, the rendering of an NoC topology selection for a particular traffic pattern and performance objective, based on the proposed novelties, is concluded in Section 8.4.

\subsection{Latency}

The unsaturated latency of the various $\mathrm{NoC}$ topologies evaluated in this work is shown in Figure 4(a) for the uniform traffic pattern, and in Figure 5(a), Figure 6(a), and Figure 7(a) for localized traffic with localization degrees of $0.25,0.50$, and 0.75 , respectively. In all four figures, the grouping of the latency results for the flat-mesh/meshX2 variants, CMesh/CMeshX2 variants, and the hierarchical mesh-mesh NoC variants is visible. This is because the different network types and their variants are similar in structure and resources. The elaboration of the results for latency analysis is performed with these groupings demonstrating similar features. 




(a) Unsaturated latency.

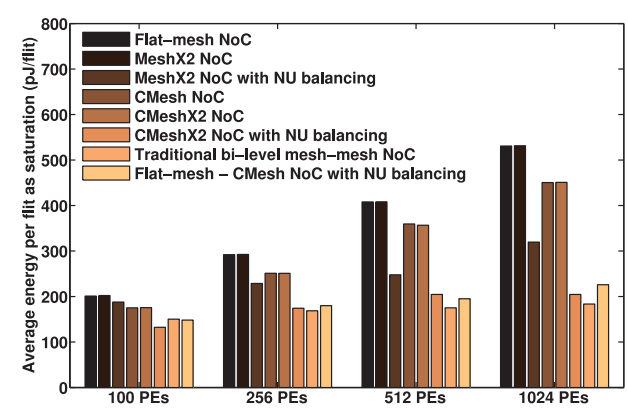

(c) Energy/flit at saturation.

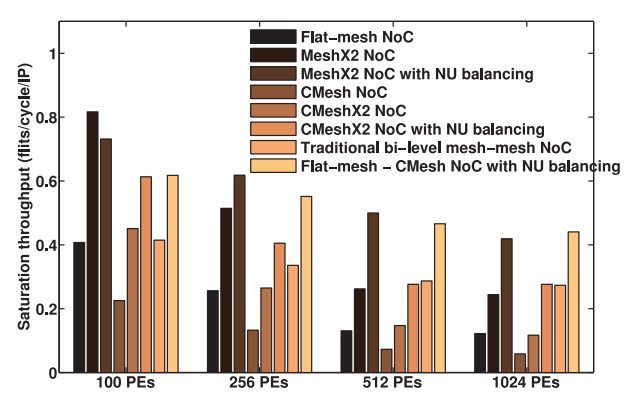

(b) Saturated throughput.

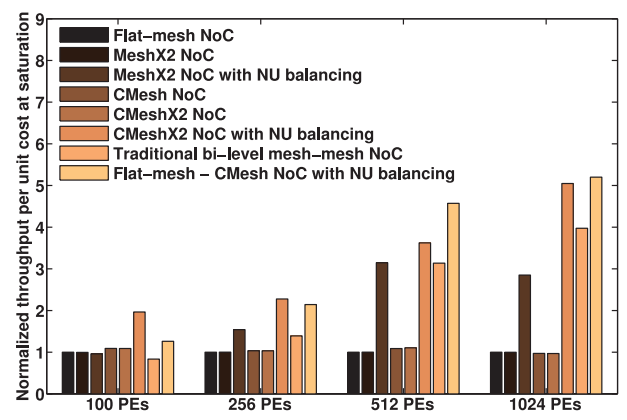

(d) Normalized throughput/cost at saturation.

Fig. 5. Network performance comparison for a localized traffic pattern (localization degree $=0.25$ )

For the NoCs evaluated in this work, the CMesh $\backslash$ CMeshX2 NoCs have the lowest unsaturated latency for the various traffic types, as they have the smallest network diameter. The flat-mesh $\backslash$ meshX2 NoCs have the highest unsaturated latency for the various traffic types due to the largest network diameter. The average unsaturated latency of the NoC with the broken hierarchy (combination of flat-mesh and CMesh) is a function of the network utilization ratio. The NoC with the broken hierarchy with a netuR $\approx 1$ has an average unsaturated latency comparable to that of the CMesh $\backslash$ CMeshX2 $(\approx 31 \%$ higher in the worst case and $\approx 15 \%$ higher in the best case for 1024 cores), but lower than that of the flat-mesh $\backslash$ meshX2 NoCs $(\approx 41 \%$ lower in the best case and $\approx 30 \%$ lower in the worst case for 1024 cores). With decreasing netUR, the average latency increases slightly but is not as high as that of the flat-mesh $\backslash$ meshX2 topologies. If the netUR is high then the unsaturated latency is similar to that of the CMesh $\backslash$ CMeshX2 topologies. This shows the importance of balancing the network utilization in heterogeneous multi-network NoC architectures (like the $\mathrm{NoC}$ with the combination of the flat-mesh and the CMesh).

\subsection{Throughput Efficiency and Energy Efficiency}

The throughput, energy/flit and the normalized throughput per unit cost at saturation for the various NoC topologies under a uniform traffic distribution are shown in Figure 4, while those under a localized traffic distribution with localization degrees of $0.25,0.50$, and 0.75 are shown in Figure 5, Figure 6, and Figure 7, respectively.

It is observed in Figures 4 through 7, that the meshX2 NoC provides the highest throughput for all network sizes under uniform traffic and for small (100 and 256 cores) network sizes under localized traffic patterns. This is expected due to the high connectivity and bandwidth provided by the two mesh networks serving each core. 


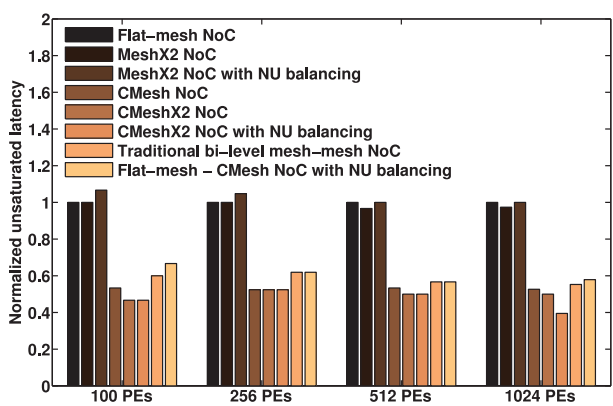

(a) Unsaturated latency.

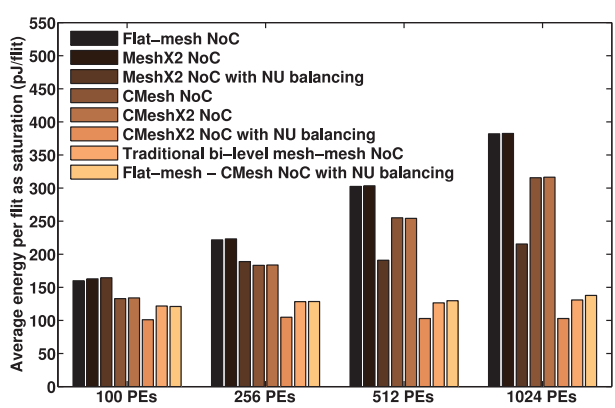

(c) Energy/flit at saturation.

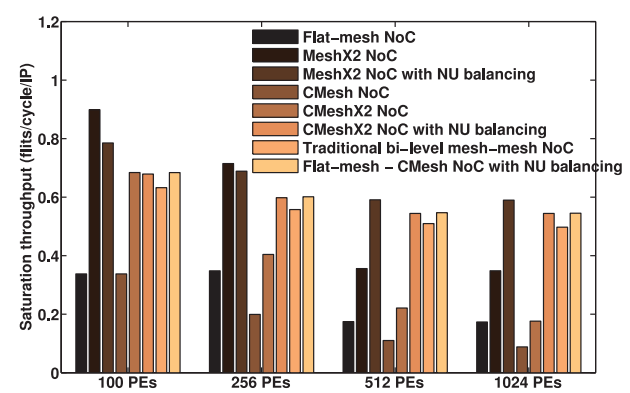

(b) Saturated throughput.

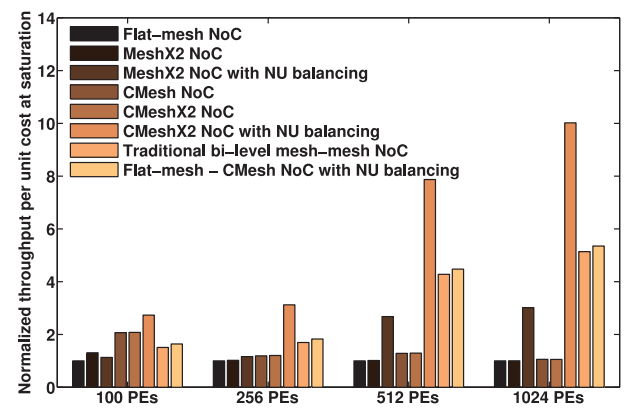

(d) Normalized throughput/cost at saturation.

Fig. 6. Network performance comparison for a localized traffic pattern (localization degree $=0.50$ ).

However, the meshX2 NoC provides a higher throughput at an additional cost in terms of the energy/flit and the total area consumed-both of which are scarce resources on a chip. Therefore, to evaluate the throughput and energy efficiency of the various NoC topologies that vary greatly in area and energy consumption, the throughput per unit cost (energy-area product) and the energy/flit are used in this work. The two notable aspects of the elaboration of the results are: (1) Collective interpretation of all performance metrics, that is, the cost of achieving the high throughput and (2) Peculiar and counterintuitive behavior of large networks similar to that observed in Das et al. [2009] and Manevich et al. [2012]. The impact of breaking the hierarchy in the traditional hierarchical NoC is discussed first followed by the impact of the network utilization balancing on a NoC with multiple networks.

8.2.1. Impact of Breaking the Hierarchy. As shown in Figure 4 for a uniform traffic pattern, the NoC topology with the broken hierarchy (labeled as "Flat-mesh-CMesh NoC with NU balancing") provides a substantially superior performance, compared to the traditional hierarchical mesh-mesh NoC (labeled as "Traditional bilevel mesh-mesh NoC"), in throughput efficiency (higher throughput per unit cost) and energy efficiency (lower energy/flit) for all network sizes. Additionally, the traditional hierarchical hard-walled mesh-mesh NoC provides the lowest throughput and energy efficiency compared to all other NoC types evaluated in this work. This highlights the benefit achievable from employing the additional links in the lower level network to break the hierarchy using the methodology proposed in this work.

At high localization degrees, for instance for a localization degree of 0.75 , as shown in Figure 7, the NoC with the broken hierarchy becomes similar in structure to the traditional hierarchical hard-walled mesh-mesh NoC (due to the small local router range). As expected, their performance becomes similar as well. The reduction in the 


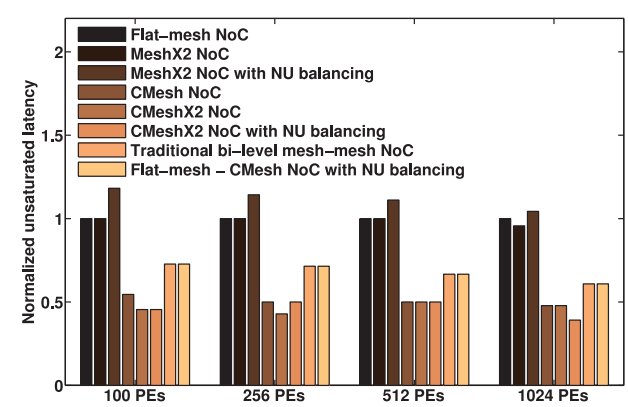

(a) Unsaturated latency.

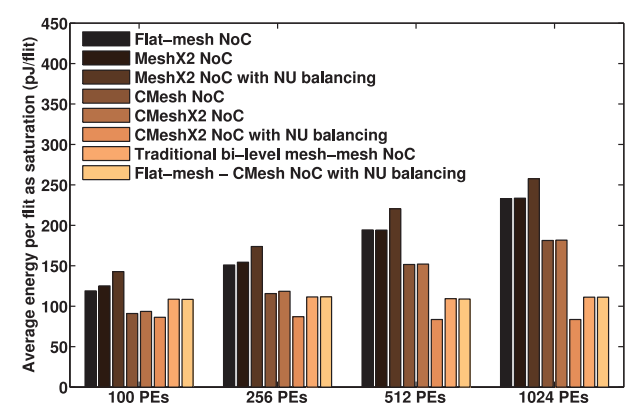

(c) Energy/flit at saturation.

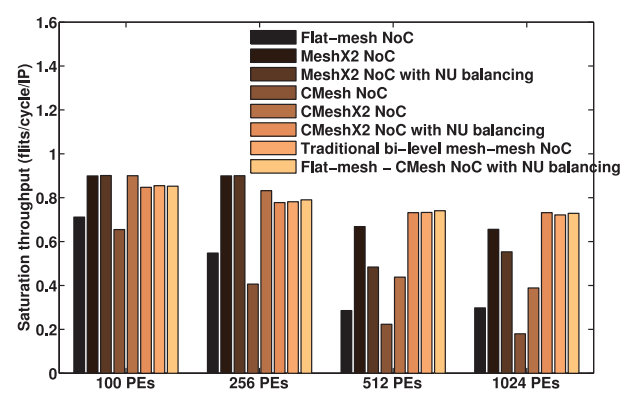

(b) Saturated throughput.



(d) Normalized throughput/cost at saturation.

Fig. 7. Network performance comparison for a localized traffic pattern (localization degree $=0.75$ )

throughput and energy efficiency for the smaller NoCs (100 and 256 cores) at high localization degrees is attributed to the higher static energy dissipation due to the under utilization of the top level network resulting from the low network utilization ratio, which is limited by the lower bound on the local router range. Therefore, if all expected workloads have very high localized traffic (localization degree greater than 0.75 ), then the performance of the NoC with the broken hierarchy and the traditional hard-walled mesh-mesh NoC is similar. However, for localized traffic patterns with localization degrees less than 0.75 (Figure 5 and Figure 6) and uniform traffic patterns (Figure 4), breaking the hierarchy provides a substantially superior performance efficiency. Further, as predicted in Kogge et al. [2008], if the exascale computing workloads are nonlocalized, then breaking the hierarchy of the traditional hierarchical hard-walled mesh-mesh NoC will provide significantly superior performance.

Additionally, breaking the hierarchy of the traditional hierarchical hard-walled NoCs provides an element of reconfigurability. Even if the future workloads have a very high localization degree, their localization radii will vary between workloads and depend on the mapping of tasks to the available hardware resources (both heterogeneous and homogeneous). In such a scenario, if a traditional hierarchical hard-walled $\mathrm{NoC}$ is used, then the localization radius is fixed by the predetermined subnet size. In this undesirable case, the workloads requiring a larger localization radius will be mapped inefficiently onto the cores. Instead, breaking the hierarchy provides the capability of defining the localization radius depending on the workload through the local router range, thereby virtually reconfiguring the subnet size at run time.

8.2.2. Impact of Locality-Aware Network Utilization Balancing on an NoC with Multiple Networks. It is observed in Figure 4 for uniform traffic and in Figures 5 and 6 for localized traffic, that while the throughput of meshX2 and CMeshX2 NoCs is twice that of the flat-mesh 
and CMesh NoCs, respectively, it is not an efficient scaling of the NoCs as indicated by the same throughput efficiency and energy efficiency in each case. Obviously, if the NoC architecture has multiple networks, as those in meshX2 and CMeshX2, the throughput increases by a factor equal to the number of networks (due to the multiple paths). Instead, a methodology similar to that employed in the NoC with the broken hierarchy for network utilization balancing needs to be employed to achieve an efficient scaling. As shown in Figures 4 through 7, employing the network utilization balancing technique on meshX2 and CMeshX2 NoCs improves the network throughput efficiency and energy efficiency. This is especially true for large network sizes under all traffic patterns evaluated in this work. The improved performance from network utilization balancing is a result of the segregation of the local and global traffic with no additional complexity added to the router design.

\subsection{Scalability Analysis}

The superior scalability of breaking the hierarchy in the mesh-mesh NoC and the application of the proposed locality-aware network utilization (NU) balancing technique compared to the counterpart NoCs not utilizing the NU balancing technique is seen in Figures 4 through 7. For instance, consider the case of the CMeshX2 NoC under a localized traffic pattern with a localization degree of 0.50, as shown in Figure 6. For clarity, the four performance metrics in the four subfigures for the CMeshX2 NoC with and without NU balancing in Figure 6, are redrawn in Figure 8. In Figure 8, the lines with circular markers trace the CMeshX2 NoC with NU balancing, whereas the lines with star markers trace the original CMeshX2 NoC without the proposed NU balancing. First, it is observed from Figure 8(a) that utilizing the NU balancing technique does not substantially affect the network latency with increasing numbers of cores. This is expected because the NU balancing technique does not alter the routing protocol, but instead only affects the network in which the packet is to be routed. Since in both cases, with and without NU balancing for the CMeshX2 NoC, the topology and the routing protocol of the network are the same, the latency is also expected to be approximately equal. However, as observed in Figure 8(b) for the saturated throughput and in Figure 8(c) for the energy/flit at saturation, the utilization of the NU balancing technique provides far superior scaling. In both cases, with and without NU balancing, the throughput decreases and the energy consumed per flit increases with scaling number of cores. Furthermore, the rate of decrease in throughput and the rate of increase in energy/flit with increasing number of cores is much lower in the case of the CMeshX2 NoC utilizing NU balancing, compared to the CMeshX2 NoC without NU balancing-both of which are desirable characteristics. Furthermore, as seen in 8 , the CMeshX2 NoC with NU balancing has a rapidly increasing trend in terms of throughput/efficiency (throughput per unit cost), which is desirable, while the CMeshX2 NoC without utilizing NU balancing has a decreasing trend, which is undesirable for scalability. This highly desirable trend of increasing throughput efficiency with increasing number of cores further emphasizes the superior scalability achievable from utilizing the NU balancing technique for the same network resources.

The data presented in Figure 8 (redrawn from Figure 6), as well as the data in Figures 4 through 7, demonstrate the energy- and performance-efficient scaling of the NoC topologies using the proposed network utilization balancing technique. The scaling is visible in scaling from 100 cores to 1024 cores, as well as the trends established in this region to anticipate continuous scaling to a number of cores higher than 1024 . Even though Figure 8 only emphasizes these scalability trends for the CMeshX2 NoC topology for the arbitrarily selected traffic pattern of localization with localization degree of 0.50 , other inspected topologies and traffic patterns also follow a similar trend. Similar data of scalability trends for different NoC topologies and traffic types 


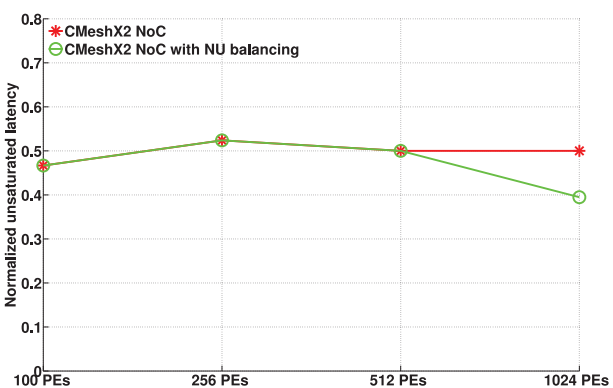

(a) Unsaturated latency.

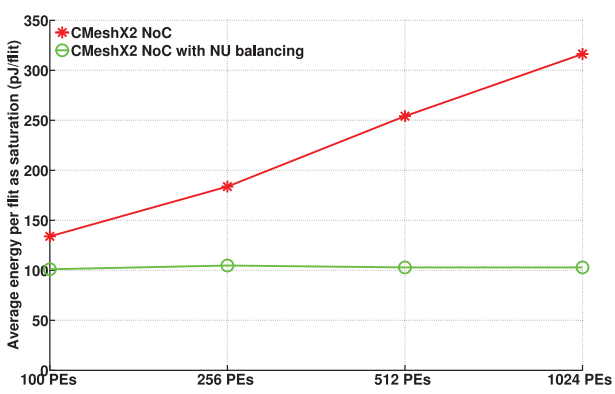

(c) Energy/flit at saturation.

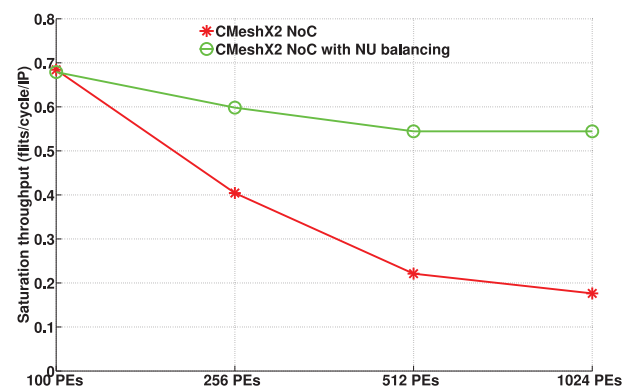

(b) Saturated throughput.

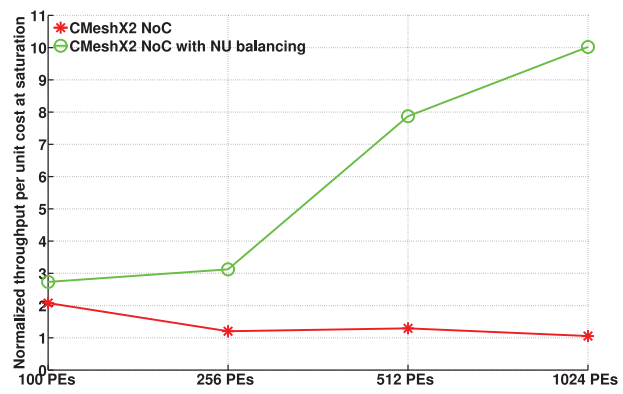

(d) Normalized throughput/cost at saturation.

Fig. 8. Two sets of data from Figure 6 are represented for the network performance scalability comparison of the CMeshX2 NoC with and without network utilization (NU) balancing. Plots are normalized to the flat-mesh NoC.

can be inferred, through visual inspection or mathematically, from the data depicted in Figures 4 through 7.

\subsection{NoC Selection for Exascale CMP Performance}

The simulation-based analysis not only provides data to validate the efficacy of the proposed network utilization balancing technique but also provides a mechanism to render a decision on the selection of an NoC type for an exascale CMP. The design of an NoC for an exascale CMP necessitates the prioritization of performance expectations between the four metrics of measurement used in this work, namely throughput, latency, energy/flit, and throughput/cost for the corresponding NoC. Note that theoretical analysis, based on small-worldness, unfortunately does not fall in agreement with the conclusions of the simulation-based results observed for these complex (hierarchical, multi-network) networks. Thus, simulation-based analysis is considered to be essential for this NoC selection process.

It is evident from the performance results depicted in Figures 4 through 7 , the performance of the NoC type depends on the network size and the expected traffic pattern. The NoCs that show the best performance for each of the metrics (highest throughput, lowest energy/flit, and highest throughput/cost) with and without employing the network utilization (NU) balancing scheme, under different traffic patterns and network sizes, are shown in Table III. The percentage difference in the performance metrics between the best NoC architecture utilizing the NU balancing technique and the best $\mathrm{NoC}$ architecture without utilizing the NU balancing scheme is also shown in Table III. In general, the network topologies employing the proposed localityaware network utilization (NU) balancing technique show the best performance (highlighted) as shown in Table III. The superior performance obtained by utilizing the 
Table III. Summary of Results for Network Performance Analysis

\begin{tabular}{|c|c|c|c|c|c|c|c|c|c|}
\hline \multicolumn{10}{|c|}{ Uniform traffic } \\
\hline \# cores & \multicolumn{3}{|c|}{ Throughput } & \multicolumn{3}{|c|}{ Energy/flit } & \multicolumn{3}{|c|}{ Throughput/cost } \\
\hline & w/ NU & w/o NU & diff & $\mathrm{w} / \mathrm{NU}$ & w/o NU & diff & $\mathrm{w} / \mathrm{NU}$ & w/o NU & diff \\
\hline 100 & meshX2 & meshX2 & $0 \%$ & hier. & CMeshX2 & $-14 \%$ & hier. & meshX2 & $36 \%$ \\
\hline 256 & meshX2 & meshX2 & $-11 \%$ & hier. & CMeshX2 & $-10 \%$ & hier. & mesh & $19 \%$ \\
\hline 512 & meshX2 & meshX2 & $-12 \%$ & hier. & CMeshX2 & $-12 \%$ & hier. & mesh & $21 \%$ \\
\hline 1024 & $\operatorname{meshX} 2$ & meshX2 & $-12 \%$ & CMeshX2 & CMeshX2 & $-29 \%$ & CMeshX2 & mesh & $56 \%$ \\
\hline
\end{tabular}

\begin{tabular}{|l||c|c|c||c|c|c||c|c|c|}
\hline \multicolumn{10}{|c|}{ Localized traffic (localization degree = 0.25) } \\
\hline $\mathbf{1 0 0}$ & meshX2 & meshX2 & $-10 \%$ & CMeshX2 & hier. & $-12 \%$ & CMeshX2 & CMesh & $80 \%$ \\
\hline $\mathbf{2 5 6}$ & meshX2 & meshX2 & $20 \%$ & CMeshX2 & hier. & $3 \%$ & CMeshX2 & hier. & $64 \%$ \\
\hline $\mathbf{5 1 2}$ & meshX2 & hier. & $74 \%$ & hier. & hier. & $11 \%$ & hier. & hier. & $46 \%$ \\
\hline $\mathbf{1 0 2 4}$ & hier. & hier. & $61 \%$ & CMeshX2 & hier. & $11 \%$ & hier. & hier. & $31 \%$ \\
\hline
\end{tabular}

\begin{tabular}{|l||c|c|c||c|c|c||c|c|c|}
\hline \multicolumn{10}{|c|}{ Localized traffic (localization degree $=\mathbf{0 . 5 0}$ ) } \\
\hline $\mathbf{1 0 0}$ & meshX2 & meshX2 & $-13 \%$ & CMeshX2 & hier. & $-17 \%$ & CMeshX2 & CMeshX2 & $32 \%$ \\
\hline $\mathbf{2 5 6}$ & meshX2 & meshX2 & $-4 \%$ & CMeshX2 & hier. & $-18 \%$ & CMeshX2 & hier. & $84 \%$ \\
\hline $\mathbf{5 1 2}$ & meshX2 & hier. & $16 \%$ & CMeshX2 & hier. & $-19 \%$ & CMeshX2 & hier. & $84 \%$ \\
\hline $\mathbf{1 0 2 4}$ & meshX2 & hier. & $19 \%$ & CMeshX2 & hier. & $-21 \%$ & CMeshX2 & hier. & $95 \%$ \\
\hline
\end{tabular}

\begin{tabular}{|l||c|c|c||c|c|c||c|c|c|}
\hline \multicolumn{10}{|c|}{ Localized traffic (localization degree = 0.75) } \\
\hline $\mathbf{1 0 0}$ & meshX2 & meshX2 & $0 \%$ & CMeshX2 & CMesh & $-5 \%$ & CMeshX2 & CMesh & $-32 \%$ \\
\hline $\mathbf{2 5 6}$ & meshX2 & meshX2 & $0 \%$ & CMeshX2 & hier. & $-22 \%$ & CMeshX2 & CMesh & $27 \%$ \\
\hline $\mathbf{5 1 2}$ & hier. & hier. & $1 \%$ & CMeshX2 & hier. & $-24 \%$ & CMeshX2 & hier. & $83 \%$ \\
\hline $\mathbf{1 0 2 4}$ & CMeshX2 & hier. & $1 \%$ & CMeshX2 & hier. & $-25 \%$ & CMeshX2 & hier. & $89 \%$ \\
\hline
\end{tabular}

NU balancing technique is further emphasized for large network sizes as shown in Table III for the best NoC topologies and in Figures 4 through 7 for all NoC topologies. Therefore, it is essential to employ such a network utilization balancing technique in order to achieve a throughput and energy-efficient scaling of the simpler network topologies for the future large CMPs and exascale computing CMPs. The preliminary results in Kemmerer and Taskin [2014] are encouraging for pursuing the idea of dynamically deciding the described local router range for improved performance in future work.

\section{SUMMARY AND CONCLUSION}

In this work, first, the hierarchy of the traditional hierarchical NoC is broken, resulting in a network with two different NoC topologies serving each core. A localityaware network utilization balancing technique is proposed to use the combination of networks efficiently in terms of throughput per unit cost and energy per flit. The locality-aware network utilization technique is also employed on multi-network NoCs, specifically the meshX2 and CMeshX2 topologies. Results of the analysis show that the proposed network utilization (NU) balancing technique is necessary for a throughputand energy-efficient scaling of the NoC architectures. For instance, for a NoC with 1024 nodes the proposed NU balancing technique provides upto 95\% higher throughput efficiency and consumes upto $29 \%$ less energy per flit compared to the best NoC topology without utilizing the NU balancing technique. Therefore, for superior scalability of the hierarchical and multi-network NoC topologies proposed in the literature, it is essential to utilize the proposed locality-aware network utilization technique in order to achieve unprecedented performance efficiency from future computing systems. 


\section{APPENDIX}

\section{A. CLUSTERING COEFFICIENTS FORMULATION}

The weighted clustering coefficient $\widetilde{C}_{i}$ equation of a node $i$ presented in (10) is repeated here for convenience to the reader:

$$
\widetilde{C}_{i}=\frac{1}{n_{i}\left(n_{i}-1\right)} \sum_{j, k} \frac{1}{\left\langle w_{i}\right\rangle} \frac{w_{i j}+w_{i k}}{2} a_{i j} a_{j k} a_{i k},
$$

where $n_{i}$ is the number of neighbors of the node $i$. The term $w_{i j}$ is the weight of the link between nodes $i$ and $j$. The term $w_{i}$ is the average value of the weights of links to node $i$, that is, $\left\langle w_{i}\right\rangle=\sum_{j}\left(w_{i j} / n_{i}\right)$. The term $a_{i j}=1$ if there is a link between nodes $i$ and $j$ and $a_{i j}=0$ otherwise.

As discussed in Section 6.2, clustering is defined pertaining to the processing element nodes in the corresponding networks. Since the nodes in each of the networks evaluated in this work have a different set of neighboring nodes and different strengths of interaction between the nodes, the weighted clustering coefficient $\widetilde{C}_{i}$ of a node $i$ defined in (10) needs to be evaluated for each of the network types separately. The network types of interest are categorized, based on the similarity of their clustering, as (1) flat-mesh $\backslash$ meshX2, (2) CMesh $\backslash$ CmeshX2, (3) traditional hierarchical meshmesh, and (4) hierarchy-broken mesh-mesh NoCs. The clustering coefficient of the flat-mesh $\backslash$ meshX2 NoC is 0 because the neighboring nodes in the flat-mesh $\backslash$ meshX2 $\mathrm{NoC}$ are not interconnected to each other.

In each of the cases of the CMesh $\backslash$ CMeshX2, the traditional hierarchical mesh-mesh $\mathrm{NoC}$ and the hierarchy broken mesh-mesh NoC, for a node $i$ belonging to a subnet or cluster $S$ with $M$ neighboring subnets or clusters the number of neighbors $n_{i}$ is given by

$$
n_{i}=\left(d_{s}-1\right)+\sum_{p \in M} d_{p},
$$

where, $d_{p}$ is the concentration degree in the case of the CMesh $\backslash$ CMeshX2 NoC or the number of nodes in the subnet. In the specific case considered in this work where all the subnets or clusters are of the same size $d$, the number of neighbors $n_{i}$ is given as

$$
n_{i}=(d-1)+M d .
$$

The clustering coefficients of the CMesh $\backslash$ CMeshX2, the traditional hierarchical meshmesh NoC and the hierarchy broken mesh-mesh NoC (heterogeneous multi-network NoC) are derived in Section A.1, Section A.2, and Section A.3, respectively, by defining the $\left\langle w_{i}\right\rangle$ term and the summation term in (10).

\section{A.1. CMesh $\backslash$ CMeshX2 NoC}

In the CMesh $\backslash$ CMeshX2 NoCs, a node $i$ consists of neighbors in its own cluster that are connected to it through a single router (a link with weight $w_{i j}=1$ ) and neighbors in the adjacent clusters that are connected to it through two routers (a link with weight $w_{i j}=1 / 2$ ). In the specific case of the same concentration degree $d$ for each router considered in this work, the $\left\langle w_{i}\right\rangle$ term is defined as

$$
\left\langle w_{i}\right\rangle=\frac{(d-1)+\frac{M d}{2}}{n_{i}} .
$$


The summation term $\sum_{j, k} \frac{w_{i j}+w i k}{2} a_{i j} a_{j k} a_{i k}$ in (10) can be broken down into several different terms as follows

$$
\sum_{j, k} x=\sum_{j, k \in S} x+M \sum_{j, k \in p} x+M \sum_{j \in S, k \in p} x+\left(\begin{array}{c}
M \\
2
\end{array}\right) \sum_{j \in p, k \in q ; p \neq q} x \quad ; p, q \in M,
$$

where,

$$
\begin{aligned}
& x=\frac{w_{i j}+w i k}{2} a_{i j} a_{j k} a_{i k} \\
& \sum_{j, k \in S}\left(\frac{w_{i j}+w i k}{2} a_{i j} a_{j k} a_{i k}\right)=\frac{(d-1)(d-2)}{2} \\
& \sum_{j, k \epsilon p}\left(\frac{w_{i j}+w i k}{2} a_{i j} a_{j k} a_{i k}\right)=\frac{d(d-1)}{4} \\
& \sum_{j \in S, k \epsilon p}\left(\frac{w_{i j}+w i k}{2} a_{i j} a_{j k} a_{i k}\right)=\frac{3 d(d-1)}{4} \\
& \sum_{j \in p, k \epsilon q ; p \neq q}\left(\frac{w_{i j}+w i k}{2} a_{i j} a_{j k} a_{i k}\right)=0 .
\end{aligned}
$$

The term $\sum_{j, k \in S}$ consists of combinations of neighbors $j$ and $k$ in the same cluster as the node $i$. The term $\sum_{j, k \in p}$ consists of combination of neighbors $j$ and $k$ both belonging to the adjacent cluster and the term $\sum_{j \in S, k \in p}$ consists of combination of neighbors $j$ belonging to the same cluster as nodes $i$ and $k$ belonging to the adjacent cluster. The term $\sum_{j \in p, k \epsilon q ; p \neq q}$ consists of combinations of neighbors in two different adjacent clusters. The last term, $\sum_{j \in p, k \in q ; p \neq q}$, is 0 because the clusters adjacent to the cluster, to which node $i$, belongs are not interconnected to each other through two or less routers, that is, $a_{j k}=0$ (the definition of a link). The clustering coefficient $C_{i}$ of a node $i$ in a CMesh $\backslash$ CMeshX2 NoC is determined by substituting (14), (15), and (16) in (10).

\section{A.2. Traditional Hierarchical Mesh-Mesh NoC}

In the case of the traditional hierarchical mesh-mesh NoC, the interaction of a node $i$ with the nodes in the adjacent subnets is similar to that of the CMesh $\backslash$ CMeshX2 NoC, that is, the nodes in adjacent subnets are connected to the node $i$ through two routers (a link with weight $w_{i j}=1 / 2$ ). However, the nodes in the same subnet as node $i$ can be separated into two different sets:

(1) a set of $n$ nodes that are adjacent to the node $i$ and are connected to $i$ through the hub at the top level and through two routers at the bottom level; and

(2) a set of $d_{s}-n-1$ nodes that are connected to the node $i$ only through the hub at the top level.

The set that is connected to the node $i$ only through the hub at the top level has a link weight $w_{i j}=1$ and the set that is connected to the node $i$ through both the hub at the top level and the router at the bottom level has a link weight $w_{i j}=3 / 2$. Using these definitions of link weights, for the specific case of equally sized subnets with $d$ nodes the term $\left\langle w_{i}\right\rangle$ term is defined as

$$
\left\langle w_{i}\right\rangle=\frac{\left(d+\frac{n}{2}-1\right)+\frac{M d}{2}}{n_{i}} .
$$


As in the case of the CMesh $\backslash$ CMeshX2 NoC discussed in Section A.1, the summation term $\sum_{j, k} \frac{w_{i j}+w i k}{2} a_{i j} a_{j k} a_{i k}$ in (10) is broken down into several different terms as follows

$$
\sum_{j, k} x=\sum_{j, k \in S} x+M \sum_{j, k \in p} x+M \sum_{j \in S, k \in p} x+\left(\begin{array}{c}
M \\
2
\end{array}\right) \sum_{j \in p, k \in q ; p \neq q} x \quad ; p, q \epsilon M,
$$

where,

$$
\begin{aligned}
& x=\frac{w_{i j}+w i k}{2} a_{i j} a_{j k} a_{i k} \\
& \sum_{j, k \in S}\left(\frac{w_{i j}+w i k}{2} a_{i j} a_{j k} a_{i k}\right)=\frac{3}{4} n(n-1)+\frac{5}{4} n(d-n-1) \\
& + \begin{cases}0, & \text { if }(d-n-1)<2 \\
\frac{1}{2}(d-n-1)(d-n-2), & \text { else }\end{cases} \\
& \sum_{j, k \in p}\left(\frac{w_{i j}+w i k}{2} a_{i j} a_{j k} a_{i k}\right)=\frac{d(d-1)}{4} \\
& \sum_{j \in S, k \in p}\left(\frac{w_{i j}+w i k}{2} a_{i j} a_{j k} a_{i k}\right)=n d+\frac{3 d(d-n-1)}{4} \\
& \sum_{j \in p, k \in q ; p \neq q}\left(\frac{w_{i j}+w i k}{2} a_{i j} a_{j k} a_{i k}\right)=0 .
\end{aligned}
$$

Each of the terms in the summation are the same as described for CMesh $\backslash$ CMeshX2 $\mathrm{NoC}$ in Section A.1. The clustering coefficient $C_{i}$ of a node $i$ in a traditional hierarchical mesh-mesh NoC can be determined by substituting (14), (17), and (18) in (10).

\section{A.3. Hierarchy Broken Mesh-Mesh NoC}

In the hierarchy-broken mesh-mesh NoC (heterogeneous multi-network NoC), the interaction of a node $i$ with its neighboring nodes depends on the location of the node $i$. If the node $i$ is not at the boundary of the subnet then its interaction with its neighbors is similar to that of the traditional hierarchical mesh-mesh NoC described in Section A.2. However, if the node $i$ is at the boundary of the subnet then the adjacent subnets can be classified into two sets:

(1) a set of $m$ subnets that share the same boundary on which the node $i$ is placed; and

(2) a set of $M-m$ subnets that do not share the same boundary on which the node $i$ is placed.

In each of the $m$ subnets, a single node interacts with the node $i$ through two hubs at the top layer and two routers at the bottom layer for a total link weight $w_{i j}=1$, while the other $d-1$ nodes interact with node $i$ only through two hubs at the top layer (link weight $w_{i j}=1 / 2$ ). The nodes in the $M-m$ subnets, that do not share the same boundary on which the node $i$ is placed, interact with node $i$ through two hubs at the top layer (link weight $w_{i j}=1 / 2$ ).

In addition to the nodes in these two sets of subnets, the interaction of the node $i$ with other nodes in the same subnet is the same as described in Section A.2 for the traditional hierarchical mesh-mesh NoC. Specifically, the nodes in the same subnet as node $i$ can be separated into two different sets:

(1) a set of $n$ nodes that are adjacent to the node $i$ and are connected to $i$ through the hub at the top level and through two routers at the bottom level; and

(2) a set of $d_{s}-n-1$ nodes that are connected to the node $i$ only through the hub at the top level. 
The set that is connected to the node $i$ only through the hub at the top level has a link weight $w_{i j}=1$. The set that is connected to the node $i$ through both the hub at the top level and the router at the bottom level has a link weight $w_{i j}=3 / 2$. Using these definitions of link weights, for the specific case of equally sized subnets with $d$ nodes, the term $\left\langle w_{i}\right\rangle$ term is defined as

$$
\left\langle w_{i}\right\rangle=\frac{\left(d+\frac{n}{2}-1\right)+\frac{m}{2}+\frac{M d}{2}}{n_{i}} .
$$

Like the other NoC topologies, the summation term $\sum_{j, k} \frac{w_{i j}+w i k}{2} a_{i j} a_{j k} a_{i k}$ in (10) can be broken down into several different terms as follows.

$$
\begin{aligned}
\sum_{j, k} x= & \sum_{j, k \epsilon S} x+(M-m) \sum_{j, k \epsilon p_{\tilde{m}}} x+(M-m) \sum_{j \epsilon S, k \in p_{\tilde{m}}} x \\
& +m \sum_{j, k \epsilon p_{m}} x+m \sum_{j \epsilon S, k \in p_{m}} x \\
& + \text { other cross adjacent subnet terms } \quad ; p_{\tilde{m}} \epsilon(M-m), p_{m} \in m,
\end{aligned}
$$

where

$$
\begin{aligned}
& \begin{array}{l}
x=\frac{w_{i j}+w i k}{2} a_{i j} a_{j k} a_{i k} \\
\begin{aligned}
\sum_{j, k \in S}\left(\frac{w_{i j}+w i k}{2} a_{i j} a_{j k} a_{i k}\right)= & \frac{3}{4} n(n-1)+\frac{5}{4} n(d-n-1)
\end{aligned} \\
\qquad \begin{array}{ll}
0, & \text { if }(d-n-1)<2 \\
\frac{1}{2}(d-n-1)(d-n-2), & \text { else }
\end{array} \\
\begin{array}{l}
\sum_{j, k \in p_{\tilde{m}}}\left(\frac{w_{i j}+w i k}{2} a_{i j} a_{j k} a_{i k}\right)=\frac{d(d-1)}{4} \\
\sum_{j \epsilon S, k \in p_{\tilde{m}}}\left(\frac{w_{i j}+w i k}{2} a_{i j} a_{j k} a_{i k}\right)=n d+\frac{3 d(d-n-1)}{4}
\end{array} \\
\sum_{j, k \in p_{m}}\left(\frac{w_{i j}+w i k}{2} a_{i j} a_{j k} a_{i k}\right)=\frac{(d-1)(d-2)}{4}+\frac{3}{4}(d-1) \\
\sum_{j \epsilon S, k \in p_{m}}\left(\frac{w_{i j}+w i k}{2} a_{i j} a_{j k} a_{i k}\right)=n(d-1)+\frac{3(d-1)(d-n-1)}{4}+\frac{5}{4} n+(d-n-1)
\end{array} \\
& \text { other cross adjacent subnet terms }=0 .
\end{aligned}
$$

Similar to the other NoC topologies, the term $\sum_{j, k \in S}$ consists of combinations of neighbors $j$ and $k$ in the same subnet as the node $i$. The term $\sum_{j, k \in p_{\tilde{m}}}$ consists of combination of neighbors $j$ and $k$, where $j$ and $k$ both belong to the adjacent subnets that do not share a boundary with the node $i$. The term $\sum_{j, k \in p_{m}}$, on the other hand, consists of adjacent subnets that do share a boundary with node $i$. Similarly, the term $\sum_{j \in S, k \epsilon p_{\tilde{m}}}$ consists of combination of neighbors $j$ belonging to the same subnet as node $i$ and $k$ belonging to the adjacent subnets that do not share a boundary with the node $i$, whereas the term $\sum_{j \in S, k \in p_{m}}$ consists of adjacent subnets that do share a boundary with node $i$. The other cross adjacent subnet terms consist of a combination of neighbors in two different adjacent subnets (either in the set $m$ or the set $M-m$ ). These cross adjacent subnet terms are 0 because the subnets adjacent to the subnet to which node $i$ belongs are not interconnected to each other through two or less routers, that is, $a_{j k}=0$ (the definition of a link). The clustering coefficient $C_{i}$ of a node $i$ in the hierarchy-broken mesh-mesh NoC is determined by substituting (14), (19), and (20) in (10). 


\section{REFERENCES}

J. Balfour and W. J. Dally. 2006. Design tradeoffs for tiled CMP on-chip networks. In Proceedings of the International Conference on Supercomputing (ICS). 187-198.

A. Barrat, M. Barthelemy, R. Pastor-Satorras, and A. Vespignani. 2004. The architecture of complex weighted networks. Proceedings of the National Academy of Sciences of the United States of America 101, 11, 37473752 .

G. B. P. Bezerra, S. Forrest, M. Forrest, A. Davis, and P. Zarkesh-Ha. 2010. Modeling NoC traffic locality and energy consumption with Rent's communication probability distribution. In Proceedings of the International Workshop on System Level Interconnect Prediction (SLIP). 3-8.

S. Borkar. 2007. Thousand core chips: A technology perspective. In Proceedings of the ACM Design Automation Conference (DAC). 746-749.

S. Bourduas and Z. Zilic. 2007. A hybrid ring/mesh interconnect for network-on-chip using hierarchical rings for global routing. In Proceedings of the International Symposium on Networks-on-Chip (NOCS). 195-204.

R. Das, S. Eachempati, A. K. Mishra, V. Narayanan, and C. R. Das. 2009. Design and evaluation of a hierarchical on-chip interconnect for next-generation CMPs. In Proceedings of the International Symposium on High Performance Computer Architecture (HPCA). 175-186.

W. Heirman, J. Dambre, D. Stroobandt, and J. Van Campenhout. 2008. Rent's rule and parallel programs: Characterizing network traffic behavior. In Proceedings of the International Workshop on System Level Interconnect Prediction (SLIP). 87-94.

A. B. Kahng, B. Li, L. Peh, and K. Samadi. 2009. ORION 2.0: A fast and accurate NoC power and area model for early-stage design space exploration. In Proceedings of the Design, Automation Test in Europe (DATE). 423-428.

J. Kemmerer and B. Taskin. 2014. Range-based dynamic routing of hierarchical on chip network traffic. In Proceedings of IEEE / ACM International Workshop on System Level Interconnect Prediction (SLIP). 1-9.

J. Kim, J. Balfour, and W. J. Dally. 2007. Flattened butterfly topology for on-chip networks. Computer Architecture Letters, 37-40.

P. Kogge, K. Bergman, S. Borkar, D. Campbell, W. Carlson, W. Dally, M. Denneau, P. Franzon, W. Harrod, K. Hill, J. Hiller, S. Karp, S. Keckler, D. Klein, R. Lucas, M. Richards, A. Scarpelli, S. Scott, A. Snavely, T. Sterling, R. S. Williams, and K. Yelick. 2008. ExaScale Computing Study: Technology Challenges in Achieving Exascale Systems. DARPA.

R. Manevich, I. Cidon, and A. Kolodny. 2012. Handling global traffic in future CMP NoCs. In Proceedings of the International Workshop on System Level Interconnect Prediction (SLIP). 40-47.

R. Marculescu, U. Y. Ogras, P. Li-Shiuan, N. E. Jerger, and Y. Hoskote. 2009. Outstanding research problems in NoC Design: System, microarchitecture, and circuit perspectives. IEEE Trans. Comput.-Aided Design Integr. Circuits Syst. 28, 1, 3-21.

U. Y. Ogras and R. Marculescu. 2006. "It's a small world after all": NoC performance optimization via longrange link insertion. IEEE Trans. Very Large Scale Integr. Syst. 14, 7, 693-706.

J. Saramäki, M. Kivelä, J. P. Onnela, K. Kaski, and J. Kertész. 2007. Generalizations of the clustering coefficient to weighted complex networks. Physical Review E, Statistical, Non-Linear and Soft Matter Physics 75, 2.

V. Soteriou, H. Wang, and L. Peh. 2006. A Statistical traffic model for on-chip interconnection networks. In Proceedings of the IEEE International Symposium on Modeling, Analysis, and Simulation (MASCOTS). 104-116.

T. Strang and C. Bauer. 2007. Context-aware elevator scheduling. In Proceedings of Advanced Information Networking and Applications Workshops (AINAW). 276-281.

H. Wang, L.-S. Peh, and S. Malik. 2003. Power-driven design of router microarchitectures in on-chip networks. In Proceedings of the IEEE/ACM International Symposium on Microarchitecture (MICRO). 105-116.

Y. J. Yoon, N. Concer, M. Petracca, and L. Carloni. 2010. Virtual channels vs. multiple physical networks: A comparative analysis. In Proceedings of the ACM Design Automation Conference (DAC). 162-165.

Received January 2013; revised July 2013, May 2014; accepted March 2015 